\title{
The effect of haptic guidance and visual feedback on learning a complex tennis task
}

\author{
Laura Marchal-Crespo $\cdot$ Mark van Raai $\cdot$ \\ Georg Rauter $\cdot$ Peter Wolf $\cdot$ Robert Riener
}

Received: 13 February 2013 / Accepted: 22 August 2013 / Published online: 8 September 2013

(C) Springer-Verlag Berlin Heidelberg 2013

\begin{abstract}
While haptic guidance can improve ongoing performance of a motor task, several studies have found that it ultimately impairs motor learning. However, some recent studies suggest that the haptic demonstration of optimal timing, rather than movement magnitude, enhances learning in subjects trained with haptic guidance. Timing of an action plays a crucial role in the proper accomplishment of many motor skills, such as hitting a moving object (discrete timing task) or learning a velocity profile (time-critical tracking task). The aim of the present study is to evaluate which feedback conditions-visual or haptic guidance-optimize learning of the discrete and continuous elements of a timing task. The experiment consisted in performing a fast tennis forehand stroke in a virtual environment. A tendon-based parallel robot connected to the end of a racket was used to apply haptic guidance during training. In two different experiments, we evaluated which feedback condition was more adequate for learning: (1) a time-dependent discrete task-learning to start a tennis stroke and (2) a tracking task-learning to follow a velocity profile. The effect that the task difficulty and subject's initial skill level have on the selection of the optimal training condition was further evaluated. Results showed that the training condition that maximizes learning of the discrete time-dependent motor task depends on the subjects' initial skill level. Haptic guidance was especially suitable
\end{abstract}

L. Marchal-Crespo $(\bowtie) \cdot$ M. van Raai · G. Rauter · P. Wolf .

R. Riener

Sensory-Motor Systems (SMS) Lab, Institute of Robotics and Intelligent Systems (IRIS), ETH Zurich, Zurich, Switzerland e-mail: laura.marchal@hest.ethz.ch

L. Marchal-Crespo $\cdot$ M. van Raai · G. Rauter $\cdot$ P. Wolf $\cdot$ R. Riener Medical Faculty, Balgrist University Hospital, University

of Zurich, Zurich, Switzerland for less-skilled subjects and in especially difficult discrete tasks, while visual feedback seems to benefit more skilled subjects. Additionally, haptic guidance seemed to promote learning in a time-critical tracking task, while visual feedback tended to deteriorate the performance independently of the task difficulty and subjects' initial skill level. Haptic guidance outperformed visual feedback, although additional studies are needed to further analyze the effect of other types of feedback visualization on motor learning of time-critical tasks.

Keywords Haptic guidance · Visual feedback · Motor learning $\cdot$ Timing task

\section{Introduction}

During robot-aided training, subjects are assisted with haptic guidance from a robotic device to move their limbs through a correct kinematic pattern. Much of the work on robot-aided training has focused on movement rehabilitation after neurological injuries (Marchal-Crespo and Reinkensmeyer 2009) while there has been little effort in training other motor skills such as athletics (Hua-wei et al. 2006; Morizono et al. 1997; Rauter et al. 2010). The possibility of using robotic devices to support training of athletic motor skills is attractive, since robots can physically assist in achieving more advanced strategies of movement that may be too dangerous or frustrating for the novel trainee, such as learning complex gymnastics moves like walking on a narrow beam (Domingo and Ferris 2010). However, there is little evidence that haptic guidance improves human motor learning when compared to unassisted practice (Wulf et al. 1998; Marchal-Crespo et al. 2010b) or to a different form of guidance, such as auditory or visual 
feedback (Sigrist et al. 2013). In fact, the guidance hypothesis in motor learning research states that physically guiding a movement will impair motor learning because it changes the input-output relationship of the task to be learned (Salmoni et al. 1984; Schmidt and Walter 1984).

Novel robot-aided training strategies have been developed based on the common clinical practice of physical therapists: they provide just enough guidance to allow participants to practice the task, while fading the guidance as training progresses to encourage learning (Emken et al. 2007; Aoyagi et al. 2007). Recent studies that employed this form of fading assistance in motor learning experiments have contradicted the guidance hypothesis. In Marchal-Crespo et al. (2010a), the authors found that training with fading haptic guidance from a robotic wheelchair trainer improved the steering ability of healthy children significantly more than training without guidance. Similarly, in Marchal-Crespo et al. (2010b), subjects who practiced with guidance from a robotic steering wheel learned to time their movements better than subjects who practiced without physical guidance. Haptic demonstration of optimal timing, rather than of movement magnitude, apparently transferred to the subjects trained with fading haptic guidance.

Recent studies indicate that haptic guidance seems to be especially useful for learning the timing components of discrete motor tasks. In a study of the effect of ski poles when learning to use a ski simulator (Wulf et al. 1998), subjects learned the timing for switching force between the feet when slalom skiing better when poles were provided. Similarly, training with haptic guidance benefited learning to play a computerized pinball-like game in especially lower-skilled subjects (Milot et al. 2010). Haptic guidance has been shown to also benefit learning to reproduce the temporal, but not spatial, characteristics of a complex spatiotemporal curve (Feygin et al. 2002). A positive effect of haptic guidance was found on the time-related components of a continuous visuomanual tracking task, such as an increase in speed and smoothness in tracking trajectories (Bluteau et al. 2008), and better learning of temporal patterns of force (Morris et al. 2007). Thus, based on these previous studies, haptic guidance may be especially suitable for learning tasks that are sensitive to motor command timing, but less sensitive to motor command amplitude.

A timing cue could also be provided in other ways rather than haptic guidance, perhaps by displaying visual or auditory feedback. The main advantage of haptic guidance is that it reduces the overall performance error, which is of great importance in tasks where errors entail safety issues. However, in motor tasks, in which safety is not a concern, training with haptic guidance may not be a good choice, since guidance changes the dynamics of the task to be learned (Salmoni et al. 1984). Several studies have shown positive effects of concurrent visual feedback (i.e., real-time visual feedback) on learning complex motor tasks (Lee et al. 1990; Shea and Wulf 1999; Todorov et al. 1997). Concurrent visual feedback has been suggested to be especially suitable in very early learning phases of complex movements (Todorov et al. 1997), because it seems to decrease the cognitive load during learning (Wulf and Shea 2002). Virtual reality simulators have a great potential to facilitate motor learning through concurrent visual feedback. In an experiment with a virtual table tennis simulator (Todorov et al. 1997), training of a specific shot with concurrent feedback-i.e., superposing the desired virtual racket and actual racket movement-improved motor learning, when compared to training with real balls under the supervision of a real trainer. However, no previous studies have evaluated the relative benefits of haptic guidance and visual feedback when training a complex time-critical tracking task with different levels of difficulty.

Haptic guidance was found to be more beneficial for less-skilled participants in some previous studies (Milot et al. 2010; Marchal-Crespo et al. 2010b). A possible explanation for this result can be found in the challenge point theory (Guadagnoli and Lee 2004). The challenge point theory states that optimal learning is achieved when the difficulty of the task is appropriate for the individual participant's level of expertise (i.e., when the challenge point is reached). Thus, providing a difficult task to less-skilled participants would result in less learning, as compared to training when the task is adjusted to be at an appropriate skill level. Likewise, providing an easy task to a proficient participant would not be predicted to improve learning, since little new information is delivered and, therefore, new skills are not acquired. Reducing the task's difficulty through haptic guidance may benefit less-skilled participants more because it makes the task appropriately challenging.

Racket, bat, and club sports (e.g., tennis, baseball, and golf) are some examples of time-critical athletics tasks. However, timing of an action plays a crucial role in the proper accomplishment of a larger range of meaningful tasks, such as hitting a moving object (discrete task), or trying to reproduce a velocity profile (time-critical tracking task). To our knowledge, no studies have evaluated the relative impact of different forms of robotic training and visual feedback on learning this important task category in a large workspace. In the present study, we seek to find which feedback condition — haptic guidance or visual feedbackoptimizes learning of the discrete and continuous elements of a timing task - a forehand tennis stroke-based on the subjects' initial skill level. In two different experiments, we evaluate which feedback condition is more adequate for each timing task category: (1) a time-dependent discrete task (learning to initiate a tennis stroke) and (2) timecritical tracking task (learning to follow a velocity profile). We further extend the study to evaluate the effect that 
task difficulty has on the selection of the optimal training condition. The hypothesis is that initially less-skilled subjects will benefit more from haptic guidance in both timecritical category tasks, since haptic guidance seems to carry more information about the task to be learned, while visual feedback may carry less information, and thus benefit more skilled participants. Haptic guidance may be suitable to train more difficult tasks also in initially more skilled subjects.

\section{Methods}

Assistive robotic tennis trainer

The tennis trainer was developed using the $r^{3}$ (reconfigurable rope robot) system, designed at ETH Zurich (Fig. 1) (von Zitzewitz et al. 2013). The $r^{3}$ system is a versatile, tendon-based robotic device. Tendon-based robots are actuated through ropes, driven from motor-actuated winches located outside of the controllable workspace. The wires are guided from the winches over deflection units fixed on the robot frame into the workspace. The $r^{3}$ system is especially suitable for motor learning of large workspace tasks with high dynamics because it can achieve high velocities (up to $9.3 \mathrm{~m} / \mathrm{s}$ ) and accelerations, and hardly disturbs visual cues during the experiments. The $r^{3}$ system was developed as a haptic device integrated in a large cave with three stereoscopic high-resolution screens that surround the viewer.

The $r^{3}$ system was designed to be reconfigurable and adaptable to a whole variety of tasks, from high-load applications [e.g., rowing (von Zitzewitz et al. 2009; Rauter et al. 2010)] to highly dynamic movements [e.g.,

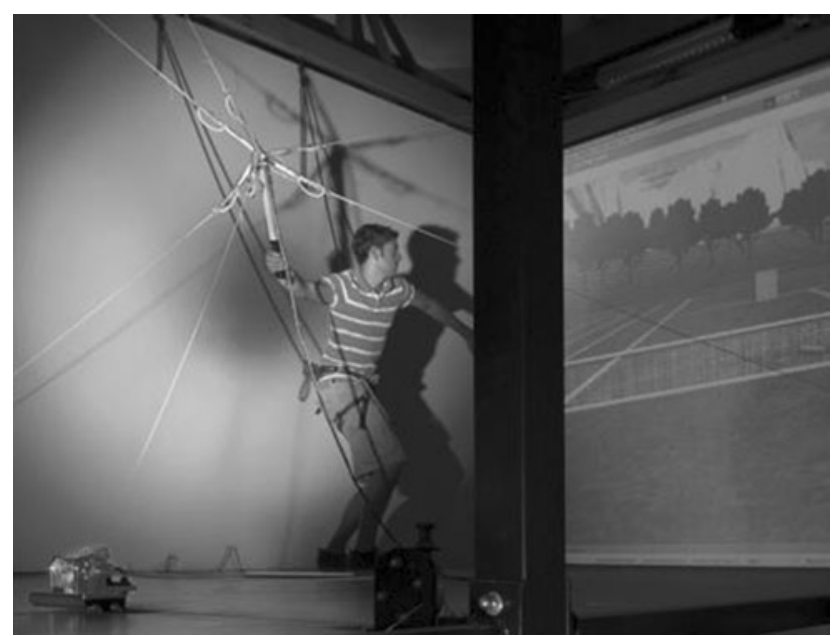

Fig. 1 The $r^{3}$ system: the reconfigurable, tendon-based robotic device developed at ETH, Zurich, for the tennis application (MarchalCrespo et al. 2012) tennis (Marchal-Crespo et al. 2012)]. Different tasks can be implemented by modifying the number and location of the deflection units. The tennis trainer presented here required a total of six ropes. Safety is crucial since subjects stood inside the cave while performing the experiment. An extensive software and hardware safety system was developed to avoid any collision between the user and the robot ropes (Marchal-Crespo et al. 2012). The subject interacting with the robot was physically restrained to a safe area with a harness and quickdraws to prevent him/her from entering the robot workspace. Furthermore, the ropes were connected to the remote end of a $0.5-\mathrm{m}$-long stick and, therefore, they were not in direct contact with the subject (Fig. 1).

The tennis task

The motor learning task consisted in a highly dynamic tennis forehand stroke performed with the right arm fully stretched. The desired stroke trajectory of the racket tip was previously measured with a 3D optical tracking system (Qualisys, Sweden). The recorded tennis stroke can be approximated by a circular arc of radius $1.1 \mathrm{~m}$ (length of the arm and racket) that subtends an angle of $1.92 \mathrm{rad}$ $\left(110^{\circ}\right)$.

One of the goals of the study was to train different velocity profiles, i.e., profiles with different maximum speeds and different locations of the speed maximum. The velocity profile should guarantee an initial and final zero velocity, and a smooth acceleration and deceleration phases. Thereby, the desired velocity at each time step was modeled following an asymmetric bell-shaped velocity profile derived from the minimum jerk equation (Flash and Hogan 1985). The minimum jerk equation was selected because it describes a well-controlled smooth profile that can be easily modified (e.g., change the maximum speed or the location of the speed maximum) by changing some parameters in the equation. The angular velocity profile ( $\mathrm{rad} / \mathrm{s})$ was derived at each sample time using the arm angle (rad) as the hand coordinate in the minimum jerk equation. The bell shape was modified to allow different slopes in the acceleration and deceleration phases, while guaranteeing smooth velocity profiles.

\section{Guidance controllers}

Different control modes were developed that allowed the robot to enforce the position and timing of the end effector (fixed haptic guidance), allowed subjects to intend the task by themselves while limiting position errors (flux guidance), and modulated the amount of guidance as training progressed (fading haptic guidance). The design and performance evaluation of the training conditions were described in detail in (Marchal-Crespo et al. 2012). Here, 
only a brief summary is given for completeness. Readers interested in the design and evaluation of the controllers are referred to (Marchal-Crespo et al. 2012) for a detailed description.

\section{Fixed haptic guidance: position controller}

The PD controller enforced the position and timing of the task. The actual end-effector position was derived from the rope lengths calculated from the encoder signals on the motor winches using forward kinematics (von Zitzewitz et al. 2013). A friction model was modeled for each drive train with a complete classical friction model (Olsson et al. 1998) and introduced in the control schema as a feed-forward term. The friction model was derived from the analyses of the static and dynamic friction responses, through empirical experimentation with each single drive train (Marchal-Crespo et al. 2012).

\section{Path controller}

Path controllers have been successfully employed in rehabilitation robotics (Duschau-Wicke et al. 2010) and sport training (Rauter et al. 2011). An artificial potential field based on the obstacle-avoidance approach described in (Khatib 1985) was designed to allow subjects to try the task by themselves. The potential field restricted the movement to a safe area surrounding the desired trajectory by correcting the movement with a repulsive potential field. The potential field could also enforce a desired positiondependent velocity profile by means of an attractive potential field. The region of influence of the repulsive potential field was delimited by a stiff tunnel and a soft tunnel that limited the area where the repulsive potential field had no influence (Marchal-Crespo et al. 2012).

Flux guidance: tunnel with assisting flux

On top of the repulsive field, a velocity flux could be added that enforced the direction and magnitude of the velocity at each trajectory point. The desired speed was calculated following a similar asymmetric bell-shaped profile as described in 2.2, but position-dependent. The assisting flux was tangent to the desired trajectory and dependent on the position of the end effector, not their time, and thus the subject was allowed to start the movement when desired. The total reactive and attractive forces were then fed into a closed-loop force controller.

No guidance along a tunnel: zero-force tunnel

The path controller was designed so that it was transparent within the tunnels when the flux was off, using a closed-loop force controller. In order to increase the transparency of the system, six miniature load cells were added in line between each rope end and the racket in order to measure the force as close to the robot the end effector as possible. The maximum force required to move the end effector inside the tunnel was less than $12 \mathrm{~N}$.

\section{Fading haptic guidance}

The fading haptic guidance controller was defined as a smooth transition between a position controller that enforces the time to start a stroke and the velocity, toward a path controller with assisting flux that enforces only the velocity. This controller was able to limit tracking errors, while constantly increasing the timing difficulty level (correct time to start the stroke) (Marchal-Crespo et al. 2012). The smooth transition was accomplished performing a weighted sum of the control signals from the position control output $f_{\text {RdesPC }}$ and from the path controller $f_{\text {RdesPath }}$. The total control signal is calculated as follows:

$f_{\text {Rdes }}=w_{1} f_{\text {RdesPC }}+w_{2} f_{\text {RdesPath }}$

The weights $w_{1}$ and $w_{2}$ were updated after each trial using a forgetting factor $f$ as in Marchal-Crespo et al. (2010a), such that at the beginning of the training session, the subjects performed with position control only $\left(w_{1}=1\right.$ and $w_{2}=0$ ).

$w_{1 i+1}(x)=f w_{1 i}$

$w_{2 i+1}(x)=1-w_{1 i+1}^{2}$

Note that the updating equations (Eq. 2) do not increase the value of $w_{2}$ at the same rate as $w_{1}$ is decreased. This is because weak position and path controllers resulted in starting the movement too late, thereby the robot tried to overcome the position error by speeding up the movement. The different updating speeds guaranteed the movement to be performed always at the same velocity profile.

Experimental protocols

The experiment was approved by the Research Ethics Committee of ETH Zurich, and all participants provided informed consent. Twenty-four healthy, right-handed adult participants (seven females) consented to take part in the experiment. Subjects were between the age of 18-34 years old (mean age $28.2 \pm 3.1$ years). The experiment took place in two different days. During the first experimental day, subjects trained to start the stroke at the correct time (a discrete timing task), while during the second day subjects learned to follow a velocity profile (a time-critical tracking task). The specific protocols for each experimental day are described in the following subsections. 


\section{Experiment 1: learning a discrete timing task}

Nineteen subjects participated in the first experimental day. A cross-over design was used to evaluate the effect of training with flux guidance, fading haptic guidance, and visual feedback on learning a discrete task. A randomized cross-over design was selected to avoid imbalances in the initial skill level between groups. The task consisted in rebounding a virtual ball moving toward them with the racket performing a forehand stroke, so that the rebounding ball hits a highlighted target area on the virtual tennis court (Fig. 2). The initial virtual position and velocity of the ball were kept constant throughout the game. The speed of the rebounding ball just after racket impact was the same as the ball speed just before impact. Three different targets located on the left (T1), middle (T2), and right (T3) side of the virtual court (Fig. 2) were randomly presented. The task difficulty depended on the target: targets on the left and middle were more challenging (range of correct racket's angles was $0.12 \mathrm{rad}$ ), while the range of correct racket's angles to hit the target on the right side was 0.27 rad. Subjects were instructed to hit as many targets as possible. To emphasize that a target was successfully hit (i.e., the ball landed on the indicated area), the highlighted target area changed the color. No other indication of movement accuracy was provided as terminal feedback.

Subjects trained with three different conditions:

1. Flux guidance: the robot was controlled with a path control with assisting flux. The controller enforced the stroke trajectory and the velocity profile, but allowed the subjects to start the movement whenever they considered adequate.

2. Fading haptic guidance: the robot started the training enforcing the correct timing to start the stroke, but faded the guidance as training progressed. The assistance was reduced during the first 35 hits ( $f=0.92$ in Eq. 2), and the last 5 hits were performed with flux guidance.

3. Concurrent visual feedback: the robot was controlled with a path control with assisting flux (flux guidance). The correct time to start the stroke was indicated by a change in the ball color (from yellow to red). In order to account for the subject reaction time, the ball changed the color $300 \mathrm{~ms}$ before the optimal time to start the movement.

Note that all conditions enforced the trajectory and velocity profile, as they were designed to train only the correct time to initiate the stroke. During training, subjects were instructed to follow along with the robot once the stroke was initiated (by themselves or the robot). However, the learning gains in the discrete task were tested in a more meaningful task: during baseline and short-term retention tests, the robot was controlled with a path controller with zero-force tunnel (no guidance along the tunnel), thereby subjects could freely perform the forehand tennis stroke without velocity restrictions. The session started with a familiarization period with flux guidance: subjects moved the robot and observed the virtual reality game. The three targets were randomly presented a total of 15 times ( 5 strokes $\times 3$ targets). During baseline, the robot was controlled with a path controller with zero-force tunnel (no guidance). The three targets were randomly presented a total of 9 times ( 3 strokes $\times 3$ target). Determination of the starting training condition was randomized. A total of 6 different configurations were possible, depending on the order of the training conditions. Subjects were randomly assigned into one of the 6 different configurations, in such a way that there were at least 3 subjects assigned at each configuration. Four subjects were trained with the configuration that started with visual feedback followed by flux guidance. Subjects were aware of which error condition they were training with. During training, subjects
Fig. 2 The virtual game projected on the front screen in experiment 1 . Three different targets located on the left, middle, and right side of the virtual court were randomly presented (here, the three targets are pictured for explanation purposes)

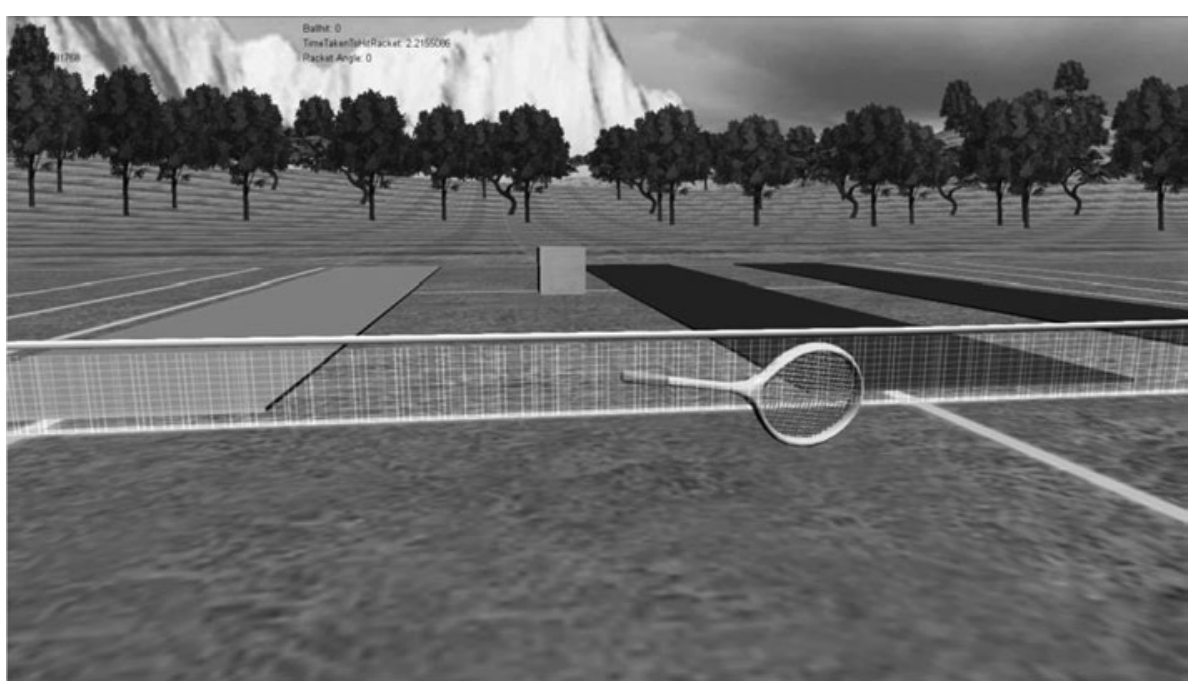


Fig. 3 The discrete task experimental protocol (day 1). $H G$ haptic guidance, $V G$ visual guidance, $F G$ flux guidance

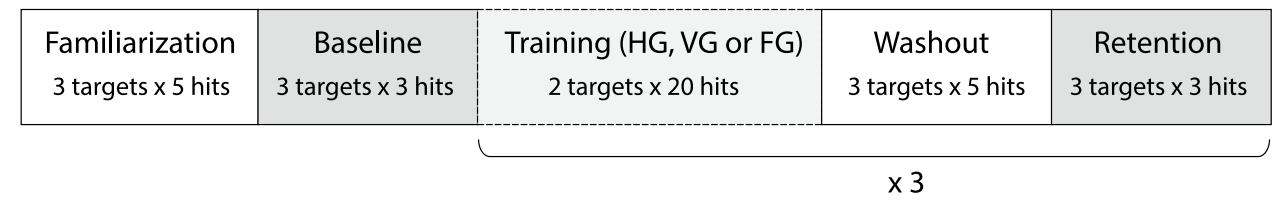

only played with the left $(T 1)$ and right $(T 3)$ targets, presented in random order a total of 40 times $(20$ hits $\times 2$ target). After training, subjects rested for $5 \mathrm{~min}$. Subjects were requested to play again with flux guidance in order to wash out possible aftereffects ( 5 strokes $\times 3$ targets). The characteristics of the short-time retention phase were identical to baseline (Fig. 3). During the retention phase, the robot was controlled with a path controller with zero-force tunnel (no guidance along the tunnel). Subjects played again with the three targets a total of 9 times ( 3 strokes $\times 3$ target). Generalization was investigated by evaluating learning to hit the untrained target $T 2$.

\section{Experiment 2: learning a time-critical tracking task}

A total of 18 participants, 13 subjects from day 1 and 5 new subjects, participated in the second experiment after at least 1 week (mean elapsed time between experiments $11.5 \pm 2.7$ days). The experiment consisted in learning to perform the tennis forehand stroke with two different velocity profiles (both different from the velocity profile performed in experiment 1). Subjects were randomly assigned to one of two groups: the haptic guidance and the visual-feedback group.

Subjects were instructed to learn two different velocity profiles (Fig. 4): a profile with a slow maximum speed

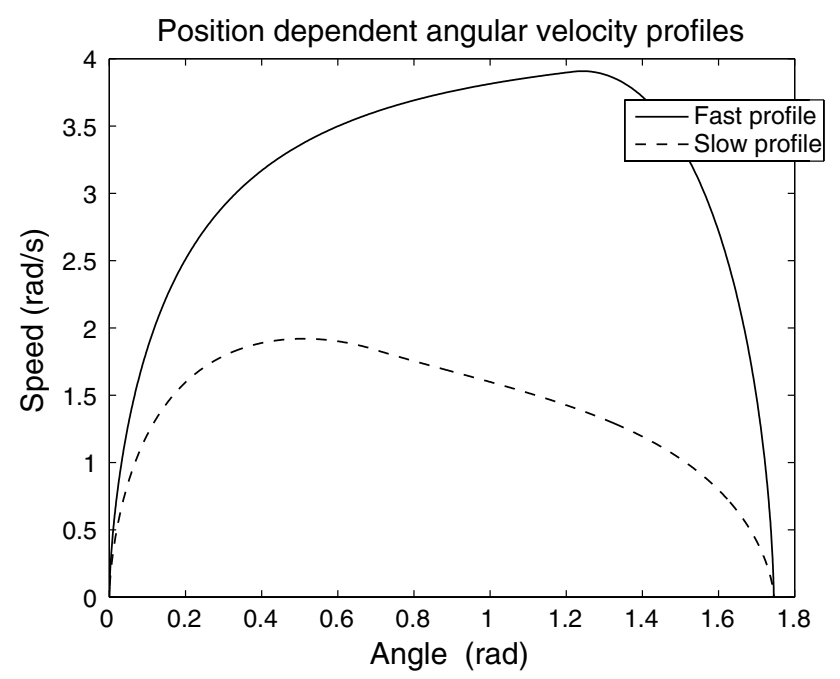

Fig. 4 The fast (solid) and slow (dashed) angular velocity profiles of the racket tip with different locations of the maximum speed to be learned $\left(v_{\max 1}=1.9 \mathrm{rad} / \mathrm{s}\right)$ located at the beginning of the stroke, and a profile with a higher maximum speed $\left(v_{\max 2}=3.9 \mathrm{rad} / \mathrm{s}\right)$ located toward the end of the stroke (Fig. 4). The fast profile was designed to be more challenging to perform than the slow one-i.e., the fast profile had a higher maximum speed value and an abrupt deceleration phase. The maximum speeds and accelerations were selected, so that subjects without any previous tennis experience could still perform the task.

Subjects were trained with two different feedback forms:

1. Haptic guidance: the robot was controlled with a position control that physically enforced the robot endeffector position and velocity. The movement of the racket was displayed as a virtual racket on the screen on the right. Subjects were instructed to always follow along the robot movement.

2. Concurrent visual feedback: the robot was controlled with a path controller with zero-force tunnel, thereby the subjects could move the racket at their selected speed inside a tunnel around the desired trajectory. Subjects were instructed to match the movement of a virtual racket displayed in diffuse pink on the right screen with the real racket displayed on the screen as a specular yellow racket (Fig. 5).

Fixed haptic guidance was selected, rather than fading haptic guidance for a fair comparison with the concurrent visual-feedback condition. Note that both conditions enforced the stroke trajectory, as they were designed to train only the velocity profile. No additional information about the movement accuracy was provided to the subjects.

The protocol started with a warm-up period in the zero-force tunnel. During baseline, subjects (regardless of the group) observed the "teacher" virtual racket performing two times the slow profile. Then, the subjects were requested to try to reproduce the movement two times. The racket movement was displayed in real time on the screen. Afterward, subjects observed two times the fast movement and were requested to reproduce the movement another two times. Afterward, the slow profile was presented once again, and subjects performed three slow profiles. Finally, the fast movement was presented once more, and the subjects performed three times the fast profile. In total, subjects performed five strokes per velocity profile. 
Fig. 5 The virtual reality scenario projected on the right side screen during training of the time-critical tracking task (experiment 2). Screenshot of the visual feedback just before a "fast" profile. Displayed in diffuse pink (dark gray) is the "teacher" racket and in specular yellow (light gray) the actual position of the racket

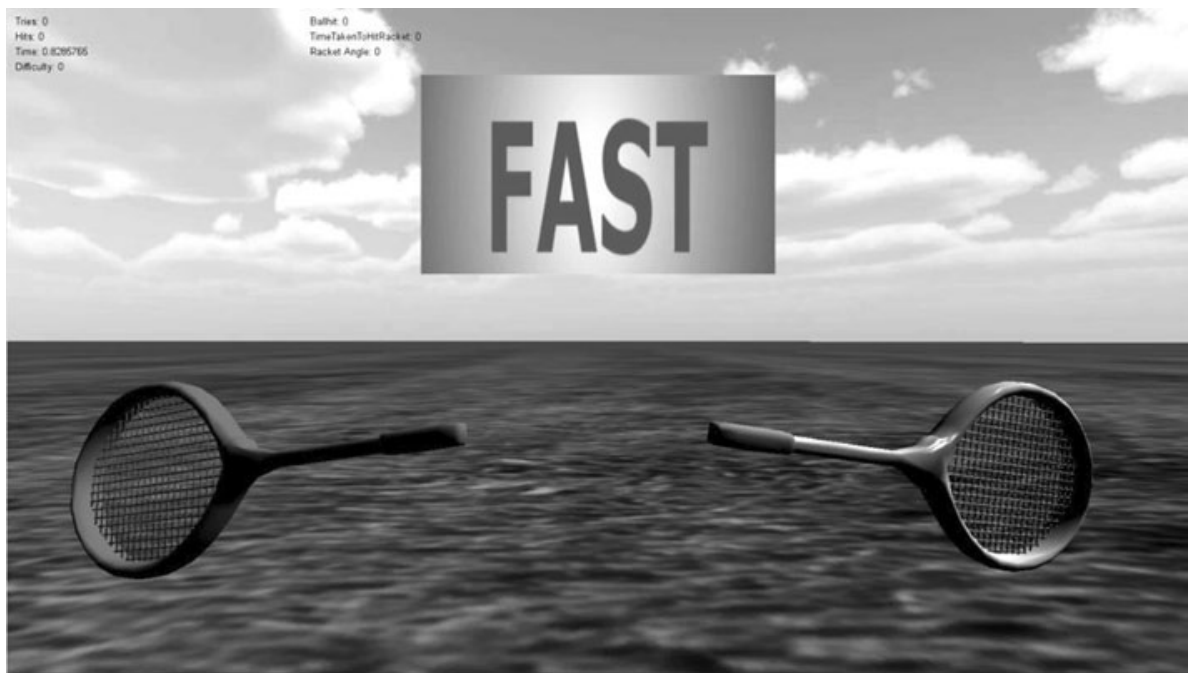

Subjects were informed about the feedback condition they were training with. They were also informed about the velocity profile of the next stroke through a message on the screen ("Fast" or "Slow" signs, Fig. 5). The velocity messages remained on the screen for $2 \mathrm{~s}$, and the subjects were informed to move as soon as the message disappeared. Subjects performed a total of 60 strokes during training, 30 per velocity profile. Each training condition included 10 catch trials (five per profile), randomly presented, for which the haptic or visual guidance was removed to avoid any slacking effect when training with the haptic condition [i.e., subjects rely on the haptic assistance (Reinkensmeyer et al. 2009)], and any possible boredom effect (i.e., performance degrades due to lack of attention). After training, a 5-min pause was provided. The short-retention phase consisted in 10 trials without feedback (five per profile) was randomly presented.

\section{Statistical analysis}

For each trial in the discrete task experiment, the absolute error, defined as the absolute value between the position of the racket at ball impact and the racket position that rebounds the ball to the center of the desired target area, was measured. The racket position at ball impact, instead of the movement time onset, was selected as performance variable. Subjects were instructed to hit as many targets as possible, while nothing was mentioned about learning the correct onset time. The accuracy feedback that subjects received during the experiment (i.e., ball landing correctly in the correct highlighted court) depended only on the position of the racket at ball impact (that was independent of the movement onset when the velocity was not enforced). Thereby, we decided to use the position of the racket at ball impact as the performance variable to be consistent with the perceived feedback.
To determine whether subjects learned, a $t$ test between baseline and retention was performed. We used a linear mixed model to test the effect that different training conditions, target difficulty, initial skill level, and order of training conditions had on the error reduction from baseline to retention phases. The error created during baseline was used as a qualitative measure of initial skill level. The model was fitted with the 3 different targets, the 3 different training conditions, and the order of the training strategies as fixed factors, the initial skill level as covariate, and the subjects as random factor. We evaluated the main effects of all factors and the interaction between the training conditions and the initial skill level, between the training conditions and the order they were performed, between the conditions and the different targets, and the 3-way interaction between the training conditions, the targets, and the initial skill level. To test the correlation between error reduction after the different training conditions and initial skill level, we performed a Pearson's correlation test. In order to examine whether the protocol design induced carryover effects (i.e., the current performance of a subject may be affected by any kind of training condition preceding the current condition), we ran a repeated measures ANOVA to compare the mean error reduction between baseline and first retention, mean error reduction between the second and first retentions, and between the third and second retentions, independently of the training conditions order.

The aim of the second experiment is to study how well subjects learn to perform a time-critical tracking task (follow a velocity profile). However, following a velocity profile is a complex task that involves learning different timings features: (1) learn the speed magnitude (e.g., learn the maximum speed), (2) learn to follow the profile timing (e.g., learn the time to produce the maximum speed), and (3) learn to follow the profile shape. Thereby, three performance variables were measured for each trial: (1) the 
absolute difference between the maximum measured speed and the desired maximum speed (i.e., maximum speed error), (2) the difference between the measured location of the speed maximum and the desired speed maximum location (i.e., speed maximum location error), and (3) the mean absolute error between the desired and performed velocity profile (i.e., mean absolute error). The position of the speed maximum was measured as the percentage of time required to achieve the maximum speed over the total time required to perform the stroke. The start (end) of a stroke was defined as the moment the stroke speed was above (below) the $10 \%$ of the maximum speed. In order to calculate the mean absolute error, the measured profile was re-sampled to match the length of the desired profile. To determine whether subjects reduced any of the performance variables after training, paired $t$ tests between baseline and retention were performed. To determine whether participating in experiment 1 affected subjects' ability to perform the tracking task, a $t$ test between subjects that participated in both experiments and novel subjects was performed using the performance variables calculated during baseline.

We used a linear mixed model to test the effect that different training conditions, velocity profiles, initial skill level, and previous knowledge of the robot (i.e., whether subjects also participated in experiment 1) had on the error created during the different experimental phases (i.e., baseline, training without catch trials, catch trials, and retention). We used a linear mixed model for each of the three performance variables. The error created during baseline was used as a qualitative measure of initial skill level. The previous knowledge of the robot factor was defined as a binary variable (subjects participated in both experiments or not). The model was fitted with the 2 different velocity profiles, the 2 training conditions, and previous knowledge of the robot as fixed factors, the initial skill level as covariate, and subjects as random factor. We evaluated the main effects of all factors and the interaction between the training conditions and the initial skill level, between the training conditions and the previous knowledge of the robot, the velocity profile and training phase, training condition and training phase, and the 3-way interaction between the training conditions, the velocity profiles and the training phases. Normal distribution was checked visually using $Q-Q$ plots. The significance value was set to $p=0.05$. Statistical analyses were performed using the SPSS and $R$ software.

\section{Results}

Experiment 1: learning a discrete timing task

Figure 6 shows the evolution of absolute error for a representative subject who performed the training conditions following the order: visual feedback + flux guidance + fading haptic guidance. Subjects learned the task with all strategies, as observed in the significant error reduction from baseline to retention ( $t$ test, visual feedback: $p=0.001$; flux guidance: $p=0.005$; fading guidance: $p=0.002$ ), with initially less-skilled subjects (i.e., subjects that created larger errors during baseline) exhibiting a larger error reduction (Fig. 7a).

A linear mixed model was employed to test the effect that the 3 different training conditions, the 3 different targets, the initial skill level-i.e., error created during baseline-and the order of training conditions had on the error reduction from baseline to retention phases. We evaluated the main effects of all factors and the interaction between the training conditions and the initial skill level, between the training conditions and the order they were performed, between the conditions and the different targets, and the 3 -way interaction between the training conditions, the targets, and the initial skill level.

The main effect of the training condition on the error reduction, independently of the initial subjects' skill level
Fig. 6 Evolution of a representative subject's error during baseline, training, and retention phases during experiment 1

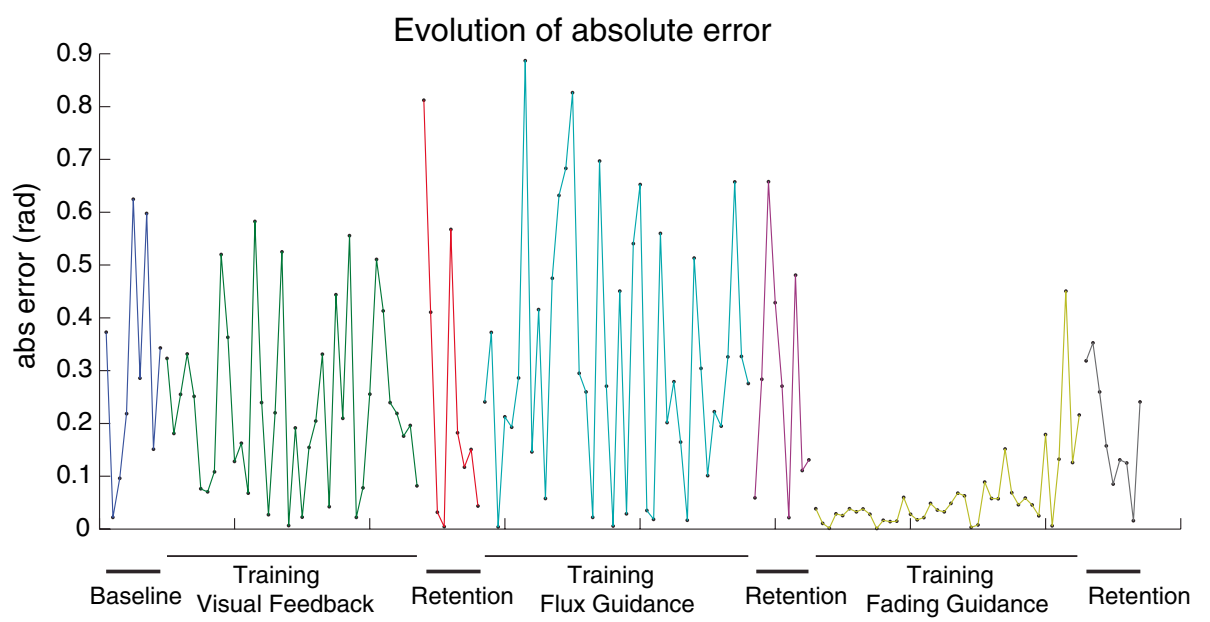



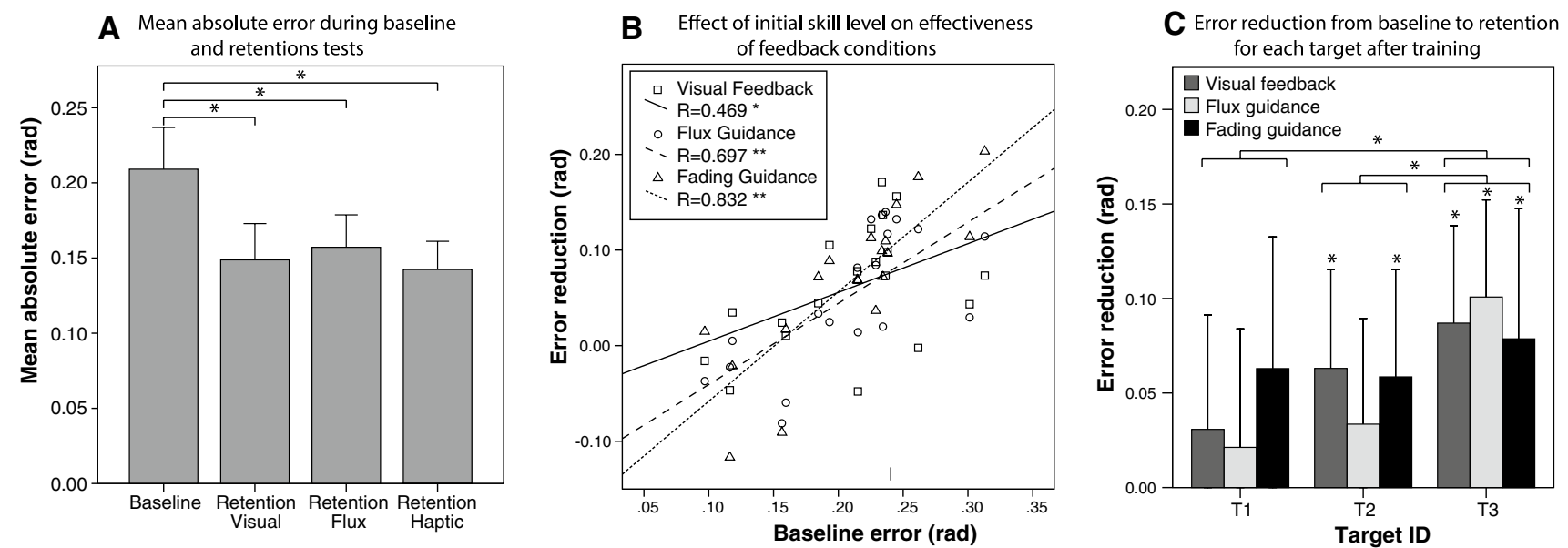

Fig. 7 Experiment 1. a Mean absolute error during baseline and retention tests. $\mathbf{b}$ effect of initial skill level (i.e., baseline error) on the error reduction after training with the different training conditions for all subjects. $\mathbf{c}$ Error reduction from baseline to retention in the trained difficult target $T 1$, the untrained target $T 2$, and the trained easy target
T3, after training with the three different training conditions. Error bars show \pm 1 SD. Bars with asterisks show a significant reduction between baseline and retention. Significant differences are identified with an asterisk $(p<0.05)$

slopes suggested that training with fading haptic guidance benefited the initially less-skilled participants, while training with visual feedback was more beneficial for initially more skilled subjects (Fig. 7b).

We found that subjects showed a tendency to reduce the errors when playing the difficult target (T1) only after playing with haptic guidance (Fig. $7 \mathrm{c}, p=0.074$ ). We also found that subjects only generalized learning after playing with visual feedback (Fig. 7c, $p=0.021$ ) and after fading haptic guidance $(p=0.044)$. Subjects learned how to play the easy target (T3) with all training conditions (visual feedback: $p=0.002$; flux guidance: $p=0.001$; fading guidance: $p=0.028)$. However, we could not find a significant interaction between training condition and targets $(p=0.206)$.

Finally, the 3-way interaction between training conditions, initial skill level, and target difficulty was nonsignificant.

We run a repeated measures ANOVA in order to examine whether the protocol design induced carryover effects. We did not find significant differences between the mean error reductions from baseline to first retention, mean error reduction from first to second retention, and from second to third retention.

\section{Experiment 2: learning a time-critical tracking task}

The $t$ tests revealed that subjects who participated in the first experiment did not show significant differences in any performance variable during baseline compared to subjects without previous knowledge of the robotic device. The previous knowledge of the robot did not affect how subjects reduced any errors, i.e., the main effect of having $R=0.832, p<0.001)$. The significant difference between
$R$. 

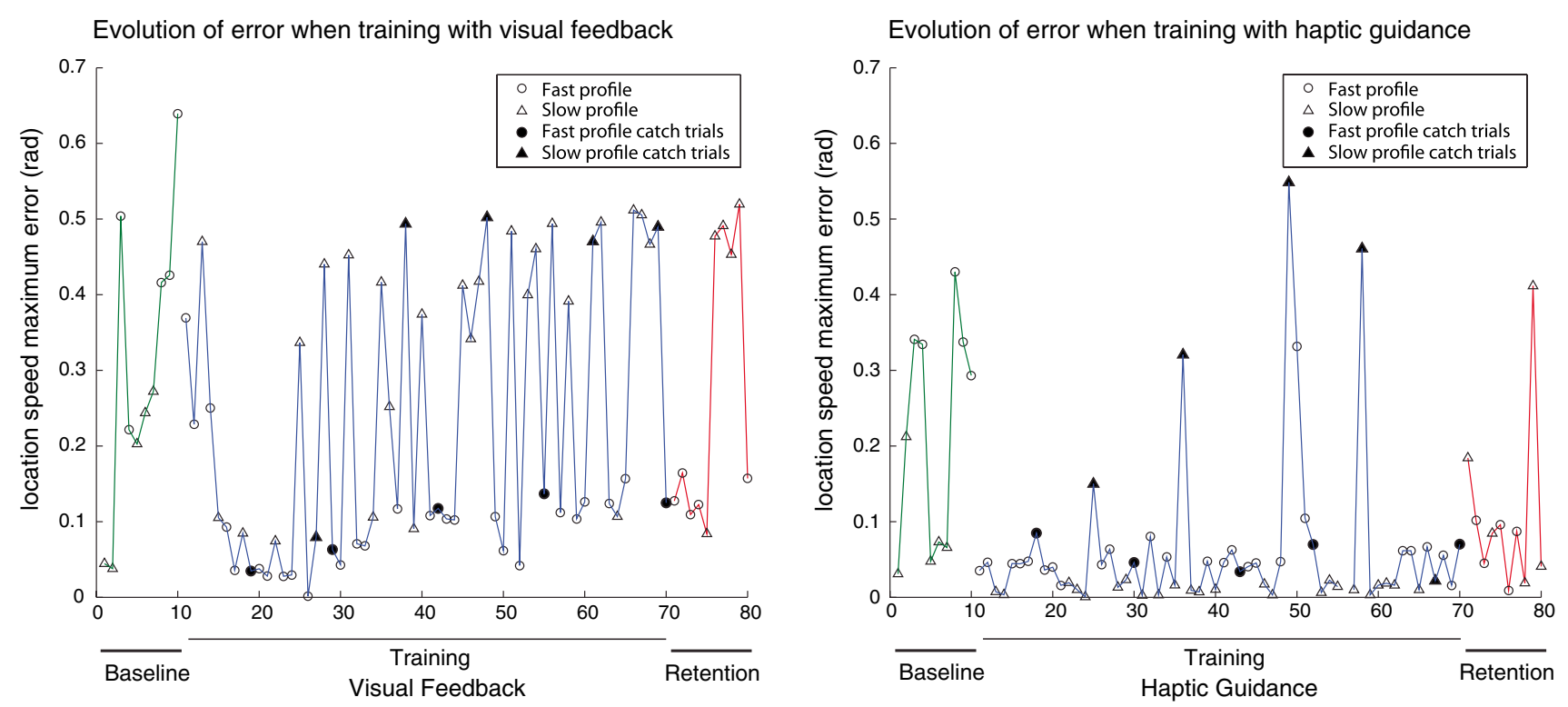

Fig. 8 Evolution of location speed maximum error during baseline, training, and retention phases of representative subjects trained with visual feedback (left), and haptic guidance (right) during experiment 2. Filled symbols represent the catch trials during training

participated also in experiment 1 was nonsignificant. The interaction of the previous knowledge of robot and the training condition was also nonsignificant.

Subjects in the visual and haptic groups did not show significant differences during baseline. Figure 8 shows the evolution of the location speed maxima error for representative subjects who trained with visual feedback (left) and haptic guidance (right).

Linear mixed models were used to test the effect that the 2 training conditions, the 2 velocity profiles, the initial skill level-i.e., error created during baseline — and the previous knowledge of the robot (i.e., whether subjects also participated in experiment 1) had on the 3 performance variables created during the different experimental phases (i.e., baseline, training without catch trials, catch trials, and retention). We added the errors created during training and catch trials into the model to have a better understanding of the subjects' performance during training with the different feedback conditions. We used a linear mixed model for each of the three performance variables. We evaluated the main effects of all factors and the interaction between the training conditions and the initial skill level, between the training conditions and the previous knowledge of the robot, the velocity profile and training phase, training condition and training phase, and the 3-way interaction between the training conditions, the velocity profiles, and the training phases.

There was a significant main effect of the training condition (mean absolute error: $p=0.002$; maximum speed error: $p=0.011$; speed maximum location error: $p=0.030$ ). We also found a significant main effect of the velocity profile on the maximum speed error $(p<0.001)$ and the speed maximum location error $(p<0.001)$. The contrast revealed that the maximum speed error in the slow profile was significantly smaller than in the fast profile. However, the speed maximum location error during the slow profile was significantly bigger than in the fast profile $(p<0.001)$.

We found a significant main effect of the training phase (i.e., baseline, training, catch trials, and retention) in all performance variables (mean absolute error: $p<0.001$; maximum speed error: $p=0.020$ with Greenhouse-Geisser correction; speed maximum location error: $p<0.001$ ). Contrast revealed that all errors were reduced during training when compared to baseline $(p<0.001)$. However, we could not find a significant difference between the errors created during baseline and retention, i.e., subjects, independently of the profile performed and the training condition, did not significantly improve after training.

We investigated the effect of the initial skill level (i.e., the error during baseline) on the effectiveness of the different training conditions and found that the interaction was nonsignificant in all the performance variables.

There was a significant interaction effect between the velocity profile and the training phase in the speed maximum location error $(p<0.001)$. Contrast revealed that subjects reduced the speed maximum location error from baseline to retention in the fast profile significantly more than in the slow profile (Fig. 10a, $p=0.007$ ). In fact, subjects significantly reduced the speed maximum location error ( $p=0.031)$, while subjects tended to degrade the error in the slow profile $(p=0.057)$.

We found a significant interaction effect between the training condition and the training phase in all performance 

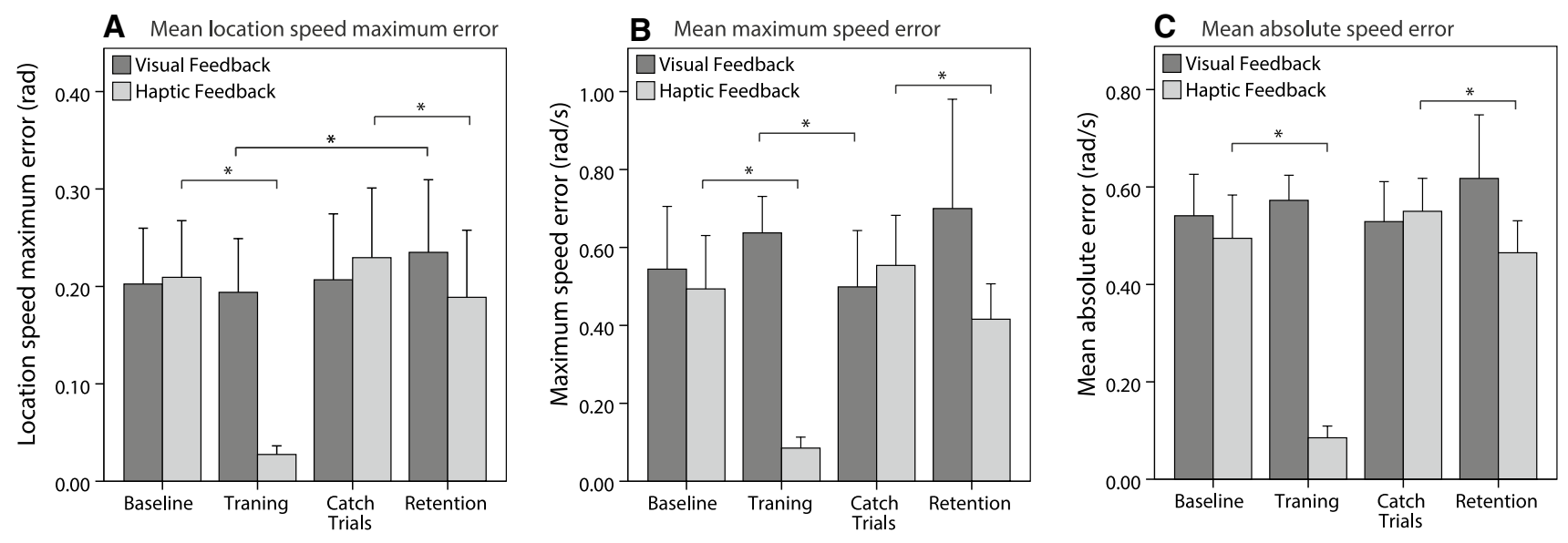

Fig. 9 Experiment 2: a mean speed maximum location error during the different training phases-baseline, training, catch trials, and retention-when training with visual feedback or haptic guidance. $\mathbf{b}$ Mean maximum speed error during the different training phases. c
Mean absolute speed error during the different training phases. Error bars show \pm 1 SD. Significantly different training phases pairs are identified with an asterisk $(p<0.05)$

experiments, we studied the effect that haptic guidance and visual feedback had on learning the discrete time-critical task of starting the tennis stroke in the correct time, and on learning the time-critical tracking motor task of following a predefined velocity profile while performing the tennis stroke. In the following subsections, we discuss in detail the results for each movement category.

The feedback condition that maximizes learning of the discrete task depends on subjects' initial skill level

Fading haptic guidance was found to enhance motor learning of the discrete time-dependent motor task, when compared to visual feedback. However, we found that the training condition-flux guidance, fading haptic guidance, and visual feedback - that maximizes learning of the discrete time-dependent motor task depended on subjects' initial skill level. Training with haptic guidance seems to benefit more the initially less-skilled subjects, while more advanced subjects seem to benefit more from visual feedback. Subjects reduced the errors significantly more when playing the easy target, apparently because non-haptically restricted training conditions did not promote learning of the more difficult targets.

A rationale for the better learning after training with fading haptic guidance can be found in the challenge point theory (Guadagnoli and Lee 2004). The challenge point theory states that optimal learning is achieved when the difficulty of the task is appropriate for the subjects' level of expertise. Thus, for initially less-skilled subjects, learning the discrete task without haptic guidance was too challenging and resulted in worse learning, as compared to training with haptic guidance. Likewise, providing haptic guidance

This study investigated the form of guidance that optimizes 
Fig. 10 Experiment 2: a speed maximum location error reduction from baseline to retention in the slow velocity profile $\left(v_{\max 1}=1.9 \mathrm{rad} / \mathrm{s}\right)$ and fast velocity profile $\left(v_{\max 1}=3.9 \mathrm{rad} / \mathrm{s}\right)$ after training with visual feedback and haptic guidance. b Speed maximum location error reduction from baseline to retention and from catch trials to retention after training with visual feedback and haptic guidance. c Maximum speed error reduction from baseline to retention and from catch trials to retention after training with visual feedback and haptic guidance. d Mean absolute error reduction from baseline to retention and from catch trials to retention after training with visual feedback and haptic guidance. Error bars show \pm 1 SD. Bars with asterisk show a significant reduction. Significantly different training condition pairs are identified with an asterisk $(p<0.05)$
A Speed maximum location error reduction from baseline to retention for different profiles
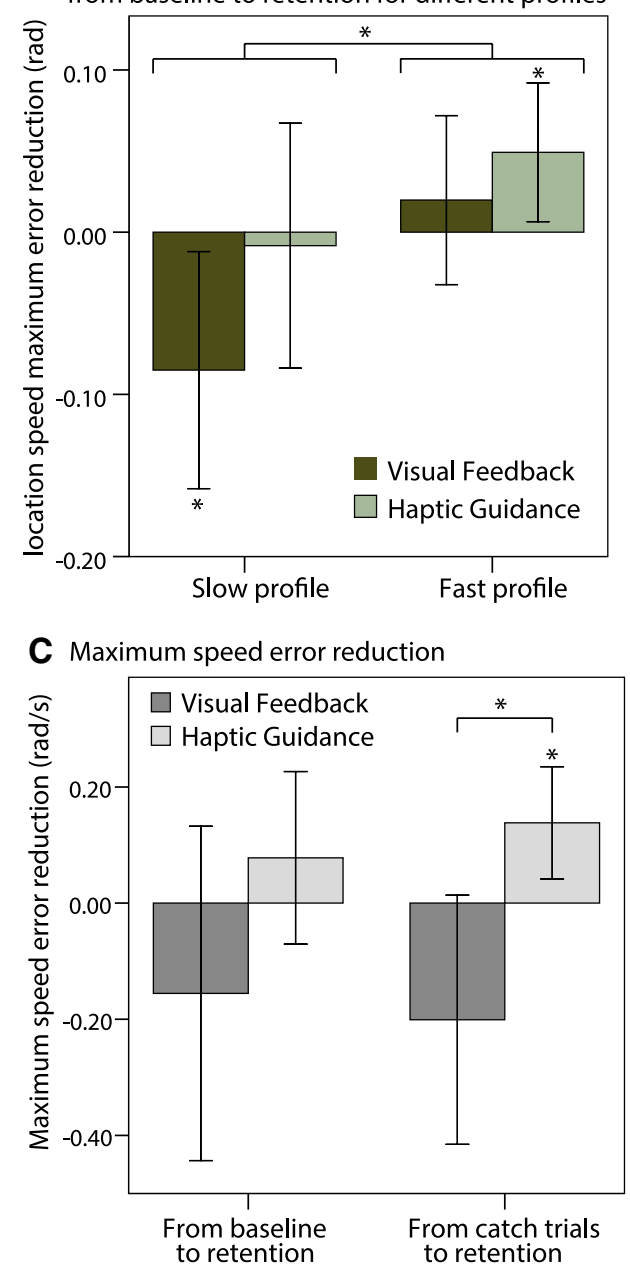

B Speed maximum location error reduction

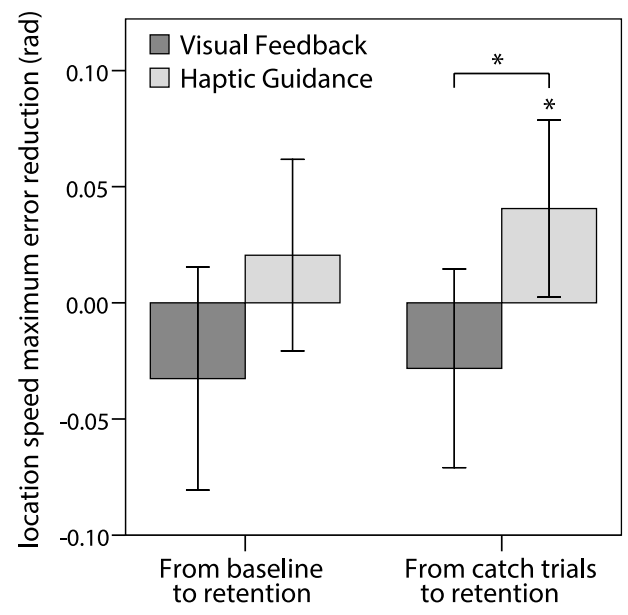

D Mean absolute error reduction

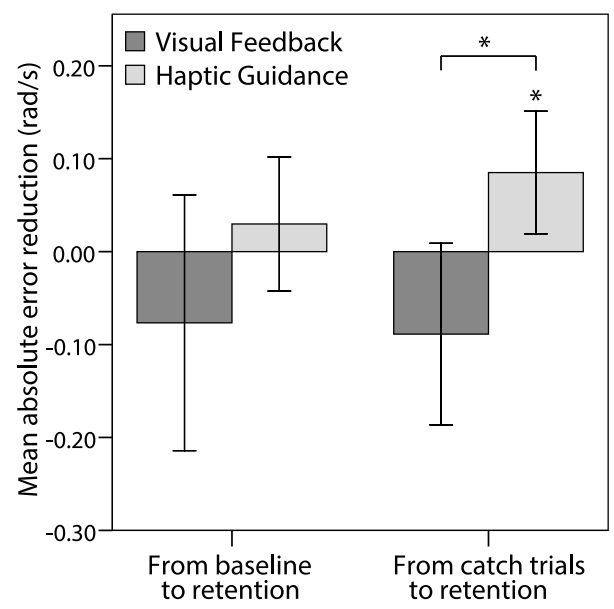

to initially more skilled subjects did not promote learning, and for some especially skilled subjects resulted in worse performance. Fading haptic guidance did not encourage any effort or attention from the more skilled subjects, and thus, new skills were not acquired. These results are in line with previous studies, which found that haptic guidance was more beneficial to learn time-related discrete tasks in initially less-skilled subjects than unassisted practice (Marchal-Crespo et al. 2010b) and error amplification (Milot et al. 2010).

Previous studies on the effect of haptic guidance on learning a discrete time-critical task were missing an important point: a timing cue could also be provided as visual or auditory feedback (Milot et al. 2010; MarchalCrespo et al. 2010b). In this paper, concurrent visual feedback was also evaluated as a training condition and results were compared to training with haptic guidance and flux guidance. In general, although training with visual feedback reduced the errors in greater extent than training with flux guidance, the difference between the two conditions was nonsignificant. The finding that concurrent visual feedback does not improve learning more than unguided training (i.e., flux guidance) contradicts the studies that found positive effects on learning complex motor tasks (Lee et al. 1990; Shea and Wulf 1999; Todorov et al. 1997). A possible rationale for the visual feedback learning limitation is that the discrete timing task was in fact a simple motor task, independently of its level of difficulty. As defined in (Wulf and Shea 2002), a motor task can be listed as a simple task if they have only one degree of freedom, can be mastered in a single practice session, and appear to be artificial. Although performing the stroke requires more than one degree of freedom, the movement initialization itself is a binary task (move or not move) and, thus, it could be defined as a simple task. Thereby, the results from our experiment are in line with studies that did not find any advantage from concurrent visual feedback when learning a simple task (Proteau 2005; Schmidt and Wrisberg 2008). Perhaps because the subjects learned to rely on the specific visual-spatial control condition required to play with visual 
feedback and failed to learn, the motor coordinates required to perform the unguided movement.

We did find a significant difference between training with haptic guidance and visual feedback: fading haptic guidance resulted in better learning than visual feedback. A significant interaction between the training conditions and initial skill level suggests that initially more skilled subjects benefit from visual feedback more than from haptic guidance, while fading haptic guidance resulted in better learning in less-skilled subjects. However, caution is needed when concluding that visual guidance seems to be especially suitable for initially more skilled subjects. From Fig. 7b, we note that the error reduction in especially advanced subjects (e.g., subjects with baseline errors smaller than $0.17 \mathrm{rad}$ ) trained with visual feedback was rather small. The lack of greater improvement may be due to a ceiling effect. The range of correct racket's angles at collision time was 0.12 rad for targets on the left and middle, and $0.27 \mathrm{rad}$ for the target on the right, thereby subjects could create small errors, but still be right on the target, failing to try to further reduce those errors. As a reference, the initially more skilled subject had a hitting success rate during baseline of $56 \%$.

Why did training with haptic guidance resulted in better learning than training with visual feedback? Visual feedback has the advantage of decreasing the cognitive load during training of especially complex tasks (Wulf and Shea 2002). We chose the ball color change as a visual cue in order to avoid subjects to focus their attention on an external visual cue. However, this type of visual feedback may have not been the most adequate to reduce the cognitive load during training, compared to training with haptic guidance. The design of the visual feedback has a great impact on the effectiveness of the visual feedback in motor learning (Sigrist et al. 2013). Thus, we cannot categorically conclude that visual feedback does not enhance motor learning. Other forms of visual cues should be systematically evaluated to determine whether concurrent visual feedback does not improve learning of discrete time-critical tasks.

Subjects reduced the errors significantly more in the easy target compared to the difficult target, probably because subjects only reduced (almost significantly) the errors in the difficult target after training with fading haptic guidance, while training with any condition resulted in learning the easy target. A possible rationale for this result can again be found in the challenge point theory (Guadagnoli and Lee 2004). Hitting the difficult target was initially too challenging. The inclusion of fading haptic guidance may have reduced the task difficulty to a more appropriate level, resulting in better motor learning.

Interestingly, training with flux guidance limited generalization-i.e., subjects did not generalize the learning gains to untrained actions. Subjects generalized to the untrained targets only after training with visual feedback and fading haptic guidance. The flux guidance condition seems to be specially limiting for novice subjects, as seen in a larger error after training with flux guidance, when compared to haptic guidance and visual feedback. This result contradicts a recent study that found that haptic guidance limited generalization in a discrete timing-based task (Marchal-Crespo and Reinkensmeyer 2008). In this previous experiment, we speculated that the limited generalization arose because subjects who received guidance experienced a narrower range of training examples. A possible rationale to this discrepancy is that, at least for initially less-skilled subjects, the tennis task was more challenging than the simpler pinball task (Marchal-Crespo and Reinkensmeyer 2008), and training with flux guidance did not result in a "rich and varied experience" (Marchal-Crespo and Reinkensmeyer 2008) that would improve learning of the untrained target. Thus, results contradicted our hypothesis: training without visual or haptic guidance was detrimental for especially less-skilled subjects.

Haptic guidance reduced performance errors after training the time-critical tracking task

In the second experiment, we found that learning to follow a desired velocity profile while performing a tennis forehand stroke was particularly challenging. Probably due to the reduced subject number and the high variability, the changes in the performance variables from baseline to retention were nonsignificant in both training groups. Although there was a tendency of better performance after training with the haptic guidance, as demonstrated by a greater reduction in error related to the maximum velocity, the location of the speed maximum, and overall mean absolute error from baseline to retention, the error reduction was non-significant. Furthermore, the visual group showed performance degradation during retention. Haptic guidance only enhanced learning of the speed maximum location, at least in the fast profile.

The lack of learning in the haptic group contradicts recent experiments on learning to perform 2D curves (Bluteau et al. 2008; Morris et al. 2007). A possible explanation to the poor learning in the current experiment may be the chosen task: the forehand stroke task required a large workspace and relatively large accelerations (and decelerations) to be performed, compared to the small task workspace and slow movements required in previous experiments. Based on the task complexity, a larger training time may have produced significant learning, at least in subjects trained with haptic guidance.

The haptic guidance worked as expected, reducing the errors when applied during training. Interestingly, subjects who trained with haptic guidance performed significantly 
better during retention than during the catch trials, while subjects in the visual-feedback group significantly increased the errors during retention. No significant differences were found between the performance errors created during baseline and during the catch trials in the visual and haptic groups, thereby suggesting that haptic guidance was better than visual guidance to retain the learning gains acquired during training.

We hypothesized a better learning in the haptic guidance group, based on previous studies that found that haptic guidance benefited learning to reproduce the temporal characteristics of a complex spatiotemporal curve (Feygin et al. 2002; Bluteau et al. 2008; Morris et al. 2007). We expected better learning especially on the fast movement (i.e., more difficult profile), because haptic guidance carries more information about the task to be learned (i.e., the kinematic structure of the movement), while visual feedback carries less information and, thus, was hypothesized to enhance learning of the slow (easy) profile. However, contrary to expectations, learning the location of the speed maximum seemed to be especially challenging in the slow profile, probably because the position maximum location was located close to the movement start. Indeed, results showed a significant greater location speed maximum error reduction from baseline to retention in the fast profile, compared to the slow profile. Although, in general, we found that the error reduction was greater after training with haptic guidance, we did not find a significant interaction between the training conditions and the speed profiles. Additionally, we did not find a significant interaction between the training condition and the initial skill level. Thereby, no conclusion can be drawn about the training condition that maximizes learning of the time-critical tracking task based on initial skill level and task difficulty.

The performance degradation of the visual-feedback group in both velocity profiles was unexpected. Several studies have shown positive effects of concurrent visual feedback on learning complex motor tasks (Lee et al. 1990; Shea and Wulf 1999; Todorov et al. 1997). It is believed that concurrent feedback can decrease the cognitive load during learning (Wulf and Shea 2002), and thus, visual feedback (provided through virtual reality simulators) has been suggested to be especially suitable to teach complex movements (Todorov et al. 1997). However, in our specific tracking task, visual feedback did not seem to reduce the cognitive load during training, as suggested by the relative large errors created during training. A possible explanation is that the type of visualization was not adequate for this special time-critical task. In fact, in trials where the visual feedback was provided, subjects seemed to be more concentrated on starting at the correct time, focusing specifically on correcting the movement when they started the stroke too late. Thus, similarly to the discrete task, caution must be taken when concluding that concurrent visual feedback does not enhance learning of continuous time-dependent tasks.

\section{Conclusion}

This study evaluated the effect of haptic guidance and visual feedback on learning the discrete and continuous elements of a tennis forehand stroke. We found that fading haptic guidance enhanced motor learning of the discrete timedependent motor task, when compared to visual feedback. Furthermore, the training condition that maximizes learning depends on subjects' initial skill level. Haptic guidance seems to be especially suitable for less-skilled subjects and for especially difficult discrete tasks, while visual feedback seems to benefit more skilled subjects. Additionally, haptic guidance also seems to promote learning in a time-critical tracking task, while visual feedback tends to deteriorate the performance, independently of the task difficulty and subjects' initial skill level. These results show that a refinement of the simplistic claim that haptic guidance benefits learning of timing tasks is needed. This refinement must describe with greater detail for which timing task category (discrete or continuous) and task difficulty haptic guidance are beneficial, based on the specific subject's initial skill level. In conclusion, although haptic guidance changes the dynamics of the task to be learned, this property does not seem to hamper learning of some specially difficult time-critical tasks in initially less-skilled subjects. Haptic guidance seems to outperform other forms of guidance, such as concurrent visual feedback, although additional studies are needed to further analyze the effect of other types of feedback visualization on motor learning of time-critical tasks. An important direction for future research is to examine long-term retention and generalization more closely following the training techniques as they have been used in this study.

Acknowledgments The authors gratefully acknowledge the contributions of Dario Wyss, Andreas Neidhart, and Michael HeroldNadig. The authors thank the Statistical Consulting service at ETH, Zurich, for their assistance in the statistical analysis. Laura Marchal-Crespo holds a Marie Curie International Income fellowship PIIF-GA-2010-272289.

\section{References}

Aoyagi D, Ichinose WE, Harkema SJ, Reinkensmeyer DJ, Bobrow JE (2007) A robot and control algorithm that can synchronously assist in naturalistic motion during body-weight-supported gait training following neurologic injury. Neural Syst Rehabil Eng IEEE Trans 15(3):387-400. doi:10.1109/TNSRE.2007.903922

Bluteau J, Coquillart S, Payan Y, Gentaz E (2008) Haptic guidance improves the visuo-manual tracking of trajectories. PLoS ONE 3(3):e1775. doi:10.1371/journal.pone.0001775 
Domingo A, Ferris D (2010) The effects of error augmentation on learning to walk on a narrow balance beam. Exp Brain Res 206(4):359-370. doi:10.1007/s00221-010-2409-x

Duschau-Wicke A, von Zitzewitz J, Caprez A, Lunenburger L, Riener R (2010) Path control: a method for patient-cooperative robotaided gait rehabilitation. Neural Syst Rehabil Eng IEEE Trans 18(1):38-48. doi:10.1109/TNSRE.2009.2033061

Emken J, Benitez R, Reinkensmeyer D (2007) Human-robot cooperative movement training: learning a novel sensory motor transformation during walking with robotic assistance-as-needed. J NeuroEng Rehabil 4(1):8

Feygin D, Keehner M, Tendick F (2002) Haptic guidance: experimental evaluation of a haptic training method for a perceptual motor skill. In: Haptic interfaces for virtual environment and teleoperator systems, 2002. HAPTICS 2002. Proceedings. 10th symposium on 2002, pp 40-47. doi:10.1109/HAPTIC.2002.998939

Flash T, Hogan N (1985) The coordination of arm movements: an experimentally confirmed mathematical model. J Neurosci 5(7):1688-1703

Guadagnoli MA, Lee TD (2004) Challenge point: a framework for conceptualizing the effects of various practice conditions in motor learning. J Mot Behav 36(2):212-224. doi:10.3200/J MBR.36.2.212-224

Hua-wei L, Tao M, Meng M (2006) Design and implementation of a fencing training robot. In: Intelligent robots and systems, 2006 IEEE/RSJ international conference on 9-15 Oct 2006, pp 36243627. doi:10.1109/IROS.2006.281716

Khatib O (1985) Real-time obstacle avoidance for manipulators and mobile robots. In: Robotics and automation. Proceedings. 1985 IEEE international conference on Mar 1985, pp 500-505. doi:10.1109/ROBOT.1985.1087247

Lee M, Moseley A, Refshauge K (1990) Effect of feedback on learning a vertebral joint mobilization skill. Phys Ther 70(2):97-102

Marchal-Crespo L, Reinkensmeyer DJ (2008) Effect of robotic guidance on motor learning of a timing task. In: Biomedical robotics and biomechatronics, 2008. BioRob 2008. 2nd IEEE RAS \& EMBS international conference on 19-22 Oct 2008, pp 199-204. doi:10.1109/BIOROB.2008.4762796

Marchal-Crespo L, Reinkensmeyer D (2009) Review of control strategies for robotic movement training after neurologic injury. J NeuroEng Rehabil 6(1):20

Marchal-Crespo L, Furumasu J, Reinkensmeyer D (2010a) A robotic wheelchair trainer: design overview and a feasibility study. J NeuroEng Rehabil 7(1):40

Marchal-Crespo L, McHughen S, Cramer S, Reinkensmeyer D (2010b) The effect of haptic guidance, aging, and initial skill level on motor learning of a steering task. Exp Brain Res 201(2):209-220. doi:10.1007/s00221-009-2026-8

Marchal-Crespo L, Rauter G, Wyss D, von Zitzewitz J, Riener R (2012) Synthesis and control of an assistive robotic tennis trainer. In: Biomedical robotics and biomechatronics (BioRob), 2012 4th IEEE RAS \& EMBS international conference on 24-27 June 2012, pp 355-360. doi:10.1109/BioRob.2012.6290262

Milot M-H, Marchal-Crespo L, Green C, Cramer S, Reinkensmeyer D (2010) Comparison of error-amplification and haptic-guidance training techniques for learning of a timing-based motor task by healthy individuals. Exp Brain Res 201(2):119-131. doi:10.1007/ s00221-009-2014-z

Morizono T, Kurahashi K, Kawamura S (1997) Realization of a virtual sports training system with parallel wire mechanism. In:
Robotics and automation, vol 3024, 1997. Proceedings, 1997 IEEE international conference on 20-25 Apr 1997, pp 30253030. doi:10.1109/ROBOT.1997.606747

Morris D, Hong T, Barbagli F, Chang T, Salisbury K (2007) Haptic feedback enhances force skill learning. In: EuroHaptics conference, 2007 and symposium on haptic interfaces for virtual Environment and Teleoperator Systems. World haptics 2007. second joint, 22-24 Mar 2007, pp 21-26. doi:10.1109/WHC.2007.65

Olsson H, Åström KJ, Canudas de Wit C, Gäfvert M, Lischinsky P (1998) Friction models and friction compensation. Eur J Control 4:176-195

Proteau L (2005) Visual afferent information dominates other sources of afferent information during mixed practice of a video-aiming task. Exp Brain Res 161(4):441-456

Rauter G, von Zitzewitz J, Duschau-Wicke A, Vallery H, Riener R (2010) A tendon-based parallel robot applied to motor learning in sports. In: Biomedical robotics and biomechatronics (BioRob), 2010 3rd IEEE RAS and EMBS international conference on IEEE, pp 82-87

Rauter G, Sigrist R, Marchal-Crespo L, Vallery H, Riener R, Wolf $P$ (2011) Assistance or challenge? Filling a gap in user-cooperative control. In: Intelligent robots and systems (IROS), 2011 IEEE/RSJ international conference on 25-30 Sept 2011, pp 3068-3073. doi:10.1109/IROS.2011.6094832

Reinkensmeyer DJ, Akoner OM, Ferris DP, Gordon KE (2009) Slacking by the human motor system: computational models and implications for robotic orthoses. In: Engineering in medicine and biology society, 2009. EMBC 2009. Annual international conference of the IEEE, pp 2129-2132

Salmoni A, Schmidt R, Walter C (1984) Knowledge of results and motor learning: a review and critical reappraisal. Psychol Bull 95(3):355-386

Schmidt RA, Walter CB (1984) Knowledge of results and motor learning: a review and critical reappraisal. Psychol Bull 95(3):355-386

Schmidt RA, Wrisberg CA (2008) Motor learning and performance: a situation-based learning approach. Human Kinetics Publishers, Cambridge

Shea CH, Wulf G (1999) Enhancing motor learning through externalfocus instructions and feedback. Hum Mov Sci 18(4):553-571

Sigrist R, Rauter G, Riener R, Wolf P (2013) Augmented visual, auditory, haptic, and multimodal feedback in motor learning: a review. Psychon Bull Rev 20(1):21-53. doi:10.3758/s13423-012-0333-8

Todorov E, Shadmehr R, Bizzi E (1997) Augmented feedback presented in a virtual environment accelerates learning of a difficult motor task. J Mot Behav 29(2):147-158

von Zitzewitz J, Rauter G, Steiner R, Brunschweiler A, Riener R (2009) A versatile wire robot concept as a haptic interface for sport simulation. In: Robotics and automation, 2009. ICRA'09. IEEE international conference on IEEE, pp 313-318

von Zitzewitz J, Morger A, Rauter G, Marchal-Crespo L, Crivelli F, Wyss D, Bruckmann T, Riener R (2013) A reconfigurable, tendon-based haptic interface for research into human-environment interactions. Robotica 31(03):441-453. doi:10.1017/ S026357471200046X

Wulf G, Shea CH (2002) Principles derived from the study of simple skills do not generalize to complex skill learning. Psychon Bull $\operatorname{Rev} 9(2): 185-211$

Wulf G, Shea CH, Whitacre CA (1998) Physical-guidance benefits in learning a complex motor skill. J Mot Behav 30(4):367-380 